ANL-HEP-PR-95-88

\title{
Breakdown of Conventional Factorization for Isolated Photon Cross Sections
}

\author{
Edmond L. Berger ${ }^{1}$, Xiaofeng Guo ${ }^{2}$, and Jianwei Qiu ${ }^{2}$ \\ ${ }^{1}$ High Energy Physics Division, Argonne National Laboratory \\ Argonne, Illinois 60439, USA \\ ${ }^{2}$ Department of Physics and Astronomy, Iowa State University \\ Ames, Iowa 50011, USA
}

(December 11, 1995)

\begin{abstract}
Using $e^{+} e^{-} \rightarrow \gamma+X$ as an example, we show that the conventional factorization theorem in perturbative quantum chromodynamics breaks down for isolated photon cross sections in a well defined part of phase space. Implications and physical consequences are discussed.

12.38.Bx, 13.65.+i, 12.38.Qk
\end{abstract}

Typeset using REVTEX 
High energy photons have long been considered an excellent probe of short-distance physics in strong interactions. They couple directly to pointlike quark constituents and do not interact much once produced. [1] Photons can also result from long-distance fragmentation of quarks and gluons, themselves produced in short-distance hard collisions. Consequently, the inclusive photon cross section at high energy includes both short-distance direct and long-distance fragmentation contributions, and the cross section is not completely perturbative. Nevertheless, in accord with the factorization theorem of perturbative quantum chromodynamics (QCD) [2], all long-distance physics associated with parton-to-photon fragmentation can be represented by non-perturbative, but well-defined and universal photon fragmentation functions, and the remainder of the theoretical expression for the cross section, calculable in QCD perturbation theory, is insensitive to the infrared region of the theory.

However, for observational reasons the inclusive cross section may not be measurable at high energy. Owing to backgrounds from, e.g., $\pi^{0} \rightarrow \gamma \gamma$, a single high energy photon is observed and the cross section is measured only when the photon is relatively isolated. In the experimental definition of isolation, a cone of half-angle $\delta$ is drawn about the direction of the photon's momentum, and the cross section is measured for photons accompanied by less than a specified amount of hadronic energy in the cone, e.g., $E_{h}^{\text {cone }} \leq E_{\max }$. Because of isolation, the experimental cross section for isolated photons depends explicitly on the isolation parameters $\delta$ and $E_{\max }$.

A proper theoretical treatment of the cross section for isolated photons requires careful consideration of the origins and cancellation of both infrared and collinear singularities in QCD perturbation theory. In a theoretical calculation, isolation of the photon restricts the final-state phase space accessible to accompanying quarks and gluons. In this Letter, using $e^{+} e^{-} \rightarrow \gamma X$ as an example, we demonstrate that this phase space restriction inevitably breaks the perfect cancellation of infrared singularities between real gluon emission and virtual gluon exchange diagrams that is required to yield finite cross sections in each perturbative order. 
Breakdown of the cancellation of infrared singularities appears first at next-to-leading order in the fragmentation contributions. The associated physics can be summarized as follows. In the fragmentation contribution, sketched in Fig. 1, hadronic energy in the isolation cone has two sources: a) energy from parton fragmentation, $E_{\text {frag }}$, and b) energy from nonfragmenting final-state partons, $E_{\text {partons }}^{\text {cone }}$, that enter the cone. When the maximum hadronic energy allowed in the isolation cone is saturated by the fragmentation energy, $E_{\max }=E_{\text {frag }}$, there is no allowance for energy in the cone from other final-state partons. In particular, if there is a gluon in the final state, the phase space for this gluon becomes restricted. By contrast, isolation does not affect the virtual gluon exchange contribution. Therefore, in the isolated case, there is a possibility that the infrared singularity from the virtual contribution may not be cancelled completely by the restricted real contribution. In the remainder of this Letter, we show that this is indeed the case, and we explore the implications.

The cross section for the inclusive yield of high energy photons in hadronic final states of $e^{+} e^{-}$annihilation is well-defined, in accord with the factorization theorem of perturbative QCD. [3] For isolated photons, we first assume that the factorization theorem holds; we then follow standard procedures to calculate the short-distance partonic hard parts perturbatively. Finally, we demonstrate that some of the hard parts have uncanceled infrared singularities.

If conventional factorization were true, the cross section for isolated photons would be expressed in the following factorized form

$$
\begin{aligned}
E_{\gamma} \frac{d \sigma_{e^{+} e^{-\rightarrow \gamma X}}^{i s o}}{d^{3} \ell} & =\sum_{c} \int_{\max \left[x_{\gamma}, \frac{1}{1+\epsilon_{h}}\right]}^{1} \frac{d z}{z} E_{c} \frac{d \hat{\sigma}_{e^{+} e^{-} \rightarrow c X}^{i s o}}{d^{3} p_{c}}\left(x_{c}=\frac{x_{\gamma}}{z}\right) \frac{D_{c \rightarrow \gamma}(z, \delta)}{z} \\
& \equiv \sum_{c} E_{c} \frac{d \hat{\sigma}_{e^{+} e^{-} \rightarrow c X}^{i s o}}{d^{3} p_{c}} \otimes D_{c \rightarrow \gamma}(z, \delta) .
\end{aligned}
$$

In Eq. (11), $x_{\gamma}=2 E_{\gamma} / \sqrt{s}, x_{c}=2 E_{c} / \sqrt{s}, \epsilon_{h}=E_{\max } / E_{\gamma}$, and the sum extends over $c=\gamma, q, \bar{q}$ and $g ; D_{c \rightarrow \gamma}(z, \delta)$ is the nonperturbative function that describes fragmentation of parton "c" into a photon. Fragmentation is assumed theoretically to be a collinear process. The lower limit of the $z$-integration results from the isolation requirement with the assumption that all fragmentation energy is in the isolation cone. [4] For the short-distance partonic hard parts, $E_{c} d \hat{\sigma}_{e^{+} e^{-} \rightarrow c X}^{i s o} / d^{3} p_{c}$, the isolation requirement is that the energy carried into the cone 
by non-fragmenting partons (i.e., partons other than $c$ ) should satisfy $E_{\text {partons }}^{\text {cone }} \leq E_{\min }(z) \equiv$ $\left[\left(1+\epsilon_{h}\right)-1 / z\right] E_{\gamma}$. To demonstrate the incomplete cancellation of infrared singularities, we present the one-loop hard part for the quark fragmentation contribution: $e^{+} e^{-} \rightarrow q X ; q \rightarrow \gamma$. The derivation of all other contributions is found in Ref. [G].

To calculate the one-loop quark fragmentation contribution, we consider $e^{+} e^{-} \rightarrow q X$ and apply Eq. (11) perturbatively to first order in $\alpha_{s}$. We derive

$$
\hat{\sigma}_{e^{+} e^{-} \rightarrow q X}^{(1) i s o}\left(x_{q}\right)=\left.\sigma_{e^{+} e^{-} \rightarrow q X}^{(1) i s o}\right|_{E_{g}\left(\text { or } E_{\bar{q}}\right) \leq E_{\min }}-\hat{\sigma}_{e^{+} e^{-} \rightarrow q^{\prime} X}^{(0) i s o}\left(x_{q}^{\prime}\right) \otimes D_{q^{\prime} \rightarrow q}^{(1)}\left(z^{\prime}\right) .
$$

The convolution over $z^{\prime}$ in Eq. (21) is the same as that in Eq. (11) but with $z$ replaced by $z^{\prime}$, and the lower limit replaced by $\max \left[x_{q}, x_{q} /\left(x_{\gamma}\left(1+\epsilon_{h}\right)\right)\right]$. In Eq. (2) $) \hat{\sigma}_{e^{+} e^{-} \rightarrow q^{\prime} X}^{(0)}\left(x_{q}^{\prime}\right)$ is the zeroth order hard part obtained from the lowest order Feynman diagram for $e^{+} e^{-} \rightarrow q \bar{q}$, and it is finite; [4] $D_{q^{\prime} \rightarrow q}^{(1)}(z)$ is the singular first order quark-to-quark fragmentation function. [3] On the right side of Eq. (2)), the first order isolated partonic cross section, $\sigma_{e^{+} e^{-} \rightarrow q X}^{(1) i s o}$, has both real gluon emission and virtual gluon exchange contributions. The real contributions are obtained from three body final-state tree diagrams $e^{+} e^{-} \rightarrow q \bar{q} g$, and the virtual contributions from one-loop interference diagrams $e^{+} e^{-} \rightarrow q \bar{q}$. Integration over the phase space of the gluon from $e^{+} e^{-} \rightarrow q \bar{q} g$ yields both infrared (when $E_{g} \rightarrow 0$ ) and collinear (when $g$ is parallel to the observed $q$ ) divergences. If factorization is valid, the infrared divergence will be cancelled by the infrared divergence from the virtual diagrams, and the collinear divergence will be cancelled by the singular second term on the right side of Eq. (22).

Our calculation of $\sigma_{e^{+} e^{-} \rightarrow q X}^{(1) i s o}$ proceeds as follows. For the real gluon emission (" $R$ ") contribution we write 四]

$$
d \sigma^{(R) i s o}=\sum_{q}\left[\frac{2}{s} F_{q}^{P C}(s)\right] e^{2} C \frac{1}{4} H d P S^{(3) i s o} .
$$

The constant $C$ is an overall color factor, $e$ is the electric charge, and the normalization factor $(2 / s) F_{q}^{P C}(s)$ includes contributions from both $\gamma^{*}$ and $Z^{0}$ intermediate states. We use dimensional regularization with $n=4-2 \epsilon$. Letting $p_{1}, p_{2}$ and $p_{3}$ label the momenta of the $q, \bar{q}$ and $g$, respectively, we derive the squared matrix element $H$. 


$$
\begin{aligned}
\frac{1}{4} H=\left(1+\cos ^{2} \theta_{1}-2 \epsilon\right) & {\left[\left(\frac{1+x_{1}^{2}}{1-x_{1}}\right) \frac{1}{y_{13}}+\frac{y_{13}}{1-x_{1}}\right.} \\
& \left.\quad-\frac{2}{1-x_{1}}-\epsilon\left(\frac{1-x_{1}}{y_{13}}+\frac{y_{13}}{1-x_{1}}+2\right)\right] \\
+ & \left(1-3 \cos ^{2} \theta_{1}\right)\left[\frac{2}{x_{1}}\left(1-\frac{y_{13}}{x_{1}}\right)\right]
\end{aligned}
$$

where $x_{1}=2 E_{1} / \sqrt{s}\left(=x_{q}\right.$ in Eq. (2) $), y_{13}=2 p_{1} \cdot p_{3} / s$, and $\theta_{1}$ is the angle between the "observed" quark and the $e^{+} e^{-}$beam axis. An overall coupling constant $\left(e \mu^{\epsilon}\right)^{2}\left(g \mu^{\epsilon}\right)^{2}$ is omitted in Eq. (田). The three particle phase space element is

$$
\begin{aligned}
d P S^{(3) i s o} & =\frac{1}{2} \frac{1}{(2 \pi)^{3}} \frac{d^{3} p_{1}}{E_{1}}\left(\frac{4 \pi}{(s / 4) \sin ^{2} \theta_{1}}\right)^{\epsilon} \frac{1}{\Gamma(1-\epsilon)} \frac{2 \pi}{s} \frac{\delta\left(x_{1}-\left(1-y_{23}\right)\right)}{x_{1}} \\
& \times \frac{s}{4}\left[\left(\frac{1}{2 \pi}\right)^{2}\left(\frac{4 \pi}{s}\right)^{\epsilon} \frac{1}{\Gamma(1-\epsilon)}\right] \delta\left(1-y_{12}-y_{13}-y_{23}\right) \frac{d y_{12}}{y_{12}^{\epsilon}} \frac{d y_{13}}{y_{13}^{\epsilon}} \frac{d y_{23}}{y_{23}^{\epsilon}} S\left(y_{12}, y_{13}, y_{23}\right)
\end{aligned}
$$

where $y_{i j}=2 p_{i} \cdot p_{j} / s$ with $i, j=1,2,3$. Function $S\left(y_{12}, y_{13}, y_{23}\right)$ in Eq. (5) specifies the constraints on the phase space due to isolation. The calculation of the isolated partonic cross section is identical to that for the inclusive cross section, except for the phase space integration. Once the $\delta$-functions are used one integral is left, say $d y_{13}$. Isolation places constraints on the limits of the $y_{13}$ integration.

In the inclusive case, the limits of integration over $\hat{y}_{13} \equiv y_{13} / x_{1}$ are from 0 to 1 . Integration of the matrix element in Eq. (田) over $\hat{y}_{13}$ from 0 to 1 generates poles in $1 / \epsilon$. As examples, a $1 / \epsilon^{2}$ singularity arises from the $1 /\left[\left(1-x_{1}\right) \hat{y}_{13}\right]$ term, and a $1 / \epsilon$ singularity from the $1 /\left(1-x_{1}\right)$ and $1 / \hat{y}_{13}$ terms. After contributions are combined from the real and the virtual gluon diagrams, all singularities cancel in the inclusive case, except one $1 / \epsilon$ term due to a collinear singularity between the fragmenting quark and the real gluon. [3] It is cancelled by the second term on the right side of Eq. (2).

In the isolated photon situation, isolation splits the integration over $\hat{y}_{13}$ into three regions:

$$
\int d \hat{y}_{13}=\int_{0}^{\min \left[\bar{y}_{c}, \bar{y}_{m}\right]} d \hat{y}_{13}+\int_{\bar{y}_{c}}^{1-\bar{y}_{c}} d \hat{y}_{13}+\int_{\max \left[\left(1-\bar{y}_{c}\right),\left(1-\bar{y}_{m}\right)\right]}^{1} d \hat{y}_{13} .
$$

Quantities $\bar{y}_{c}$ and $\bar{y}_{m}$ are

$$
\bar{y}_{c} \equiv \frac{\left(1-x_{1}\right) \sin ^{2}(\delta / 2)}{1-x_{1} \sin ^{2}(\delta / 2)} \Rightarrow\left(1-x_{1}\right) \frac{\delta^{2}}{4}
$$




$$
\bar{y}_{m} \equiv z\left(1+\epsilon_{h}\right)-\frac{1}{x_{1}}=z\left[\left(1+\epsilon_{h}\right)-\frac{1}{x_{\gamma}}\right],
$$

with $z=x_{\gamma} / x_{1}$. For the first interval in Eq. (6), the condition $0 \leq \hat{y}_{13} \leq \bar{y}_{c}$ ensures that a gluon is in the isolation cone of the fragmenting quark; and $\hat{y}_{13} \leq \min \left[\bar{y}_{c}, \bar{y}_{m}\right]$ ensures that the total hadronic energy in the isolation cone is less than $E_{\max }=\epsilon_{h} E_{\gamma}$. Similarly, the condition $\max \left[\left(1-\bar{y}_{c}\right),\left(1-\bar{y}_{m}\right)\right] \leq \hat{y}_{13} \leq 1$ for the third interval ensures that the antiquark is in the isolation cone of the fragmenting quark, and that the total hadronic energy in the isolation cone is less than $E_{\max }$. The second interval represents the situation when neither gluon nor antiquark is in the isolation cone.

Equations (6) and (7) show that the isolated cross section is identical to the inclusive cross section if $\delta=0$ or $\bar{y}_{c} \leq \bar{y}_{m}$. However, if $\bar{y}_{c}>\bar{y}_{m}$, the phase space of the final state gluon (and/or antiquark) is smaller.

We now examine in turn two separate situations: $x_{\gamma} \leq 1 /\left(1+\epsilon_{h}\right)$ and $x_{\gamma}>1 /\left(1+\epsilon_{h}\right)$. Both yield isolated photon partonic cross sections that are infrared sensitive. When $x_{\gamma} \leq$ $1 /\left(1+\epsilon_{h}\right), \bar{y}_{m} \leq 0$, and only the second interval in Eq. (6) survives. We reexpress it as

$$
\int_{\left(1-x_{1}\right) \delta^{2} / 4}^{1-\left(1-x_{1}\right) \delta^{2} / 4} d \hat{y}_{13}=\int_{0}^{1} d \hat{y}_{13}-\int_{0}^{\left(1-x_{1}\right) \delta^{2} / 4} d \hat{y}_{13}-\int_{1-\left(1-x_{1}\right) \delta^{2} / 4}^{1} d \hat{y}_{13}
$$

where $\bar{y}_{c}$ is expanded to order $\delta^{2}$. The first term on the right side of Eq. (8) is, by definition, the complete real contribution to the inclusive partonic cross section. When it is combined with the virtual contribution, as in the inclusive case, all pole terms cancel, except for one $1 / \epsilon$ term due to the collinear singularity, discussed above. The second and third terms diminish the real contribution in isolated case. The third term is innocuous in that it does not generate a $1 / \epsilon$ singularity; it yields only terms which vanish as $\delta^{2} \rightarrow 0$. However, the second term generates a number of pole terms in $1 / \epsilon$.

Neglecting all terms of $O\left(\delta^{2}\right)$ or higher, we obtain the isolated partonic cross section after including real gluon contributions from all three terms in Eq. (8) and the virtual gluon contribution. 


$$
\begin{aligned}
& E_{1} \frac{d \sigma_{e^{+} e^{-} \rightarrow q X}^{(1) i s o}}{d^{3} p_{1}}=\left[\frac{2}{s} F^{P C}(s)\right]\left[\alpha_{e m}^{2} N_{c} \frac{1}{x_{1}}\right] C_{F}\left(\frac{\alpha_{s}}{2 \pi}\right) \\
& \times\left\{( 1 + \operatorname { c o s } ^ { 2 } \theta _ { 1 } ) \left[-\left(1+x_{1}^{2}\right)\left(\frac{\ell n\left(1-x_{1}\right)}{1-x_{1}}\right)_{+}\right.\right. \\
& \quad-\frac{1+x_{1}^{2}}{\left(1-x_{1}\right)_{+}} \ell n\left(\frac{\delta^{2}}{4}\right)-\frac{3}{2} \frac{1}{\left(1-x_{1}\right)_{+}}-\frac{1}{2}\left(x_{1}-3\right) \\
&\left.+\delta\left(1-x_{1}\right)\left[\frac{2 \pi^{2}}{3}-\frac{9}{2}-\frac{1}{2} \ell n^{2}\left(\frac{\delta^{2}}{4}\right)\right]\right] \\
&\left.+\left(1-3 \cos ^{2} \theta_{1}\right)\right\} \\
& {\left[\frac{2}{s} F^{P C}(s)\right]\left[\alpha_{e m}^{2} N_{c}\left(\frac{4 \pi \mu^{2}}{(s / 4) \sin ^{2} \theta_{1}}\right)^{\epsilon} \frac{1}{\Gamma(1-\epsilon)} \frac{1}{x_{1}}\right] } \\
& \times\left(1+\cos ^{2} \theta_{1}-2 \epsilon\right) C_{F}\left(\frac{\alpha_{s}}{2 \pi}\right)\left[\left(\frac{4 \pi \mu^{2}}{s}\right)^{\epsilon} \frac{1}{\Gamma(1-\epsilon)}\right] \\
& \times\left\{\frac{1}{\epsilon^{2}}+\frac{1}{\epsilon}\left(\frac{3}{2}-\ell n \frac{\delta^{2}}{4}\right)\right\} \delta\left(1-x_{1}\right) .
\end{aligned}
$$

As explained above, the uncanceled poles in Eq. (9) come from the interval specified by the second term in Eq. (8). The singularities corresponding to these poles are infrared in nature and, as expected, are proportional to $\delta\left(1-x_{1}\right)$. These poles would be irrelevant if $x_{1} \neq 1$. However, $x_{1} \equiv x_{\gamma} / z$, and $x_{1}=1$ is kinematically allowed when $x_{\gamma}=1 /\left(1+\epsilon_{h}\right)$. Since the subtraction term in Eq. (2) vanishes when $x_{\gamma} \leq 1 /\left(1+\epsilon_{h}\right)$, the poles in Eq. (9) become the uncanceled infrared singularities of the short-distance hard part, $\hat{\sigma}_{e^{+} e^{-} \rightarrow q X}^{(1) i s o}$ defined in Eq. (2).

Having examined the situation for $x_{\gamma} \leq 1 /\left(1+\epsilon_{h}\right)$, we next consider $x_{\gamma}>1 /\left(1+\epsilon_{h}\right)$, or, equivalently, $\bar{y}_{m}>0$. The integration over $\hat{y}_{13}$ defined in Eq. (6) can be rewritten as

$$
\int d \hat{y}_{13}=\int_{0}^{1} d \hat{y}_{13}-\int_{\bar{y}_{m}}^{\bar{y}_{c}} d \hat{y}_{13}-\int_{1-\bar{y}_{c}}^{1-\bar{y}_{m}} d \hat{y}_{13}
$$

When $x_{\gamma}>1 /\left(1+\epsilon_{h}\right)$, the subtraction term on the right of Eq. (2) no longer vanishes. Combining the real contribution from the first term in Eq. (10), the virtual contribution, and the subtraction term in Eq. (2), we obtain the complete partonic inclusive cross section. (3) The third term in Eq. (10) yields contributions that vanish when $\delta^{2} \rightarrow 0$. Again, the $\underline{\text { second }}$ term generates both infrared and collinear sensitivity. (The reason that a finite 
contribution is present when $x_{\gamma}>1 /\left(1+\epsilon_{h}\right)$ is that there is a mismatch between the cone definition at the level of Feynman diagrams and collinear fragmentation through parton-tophoton fragmentation functions.) Integration yields results such as $(1 / \epsilon) \delta\left(1-x_{1}\right)$ from $1 /(1-$ $\left.x_{1}\right)^{1+\epsilon}$ terms, and $\ell n\left(\bar{y}_{m}\right)$ from $1 / y_{13}$ in Eq. (田). The $\ell n\left(\bar{y}_{m}\right)$ contribution is logarithmically divergent when $x_{\gamma} \rightarrow 1 /\left(1+\epsilon_{h}\right)$.

Because uncanceled infrared poles are present in the partonic hard parts, $\hat{\sigma}_{e^{+} e^{-} \rightarrow q X}^{(1) i s}$, we conclude that the conventional factorization theorem for the cross section of isolated photons in $e^{+} e^{-}$annihilation breaks down when $x_{\gamma} \sim 1 /\left(1+\epsilon_{h}\right)$. The value of $\epsilon_{h}$ is chosen in individual experiments. We turn now to a discussion of the implications and physical consequences.

First, we caution that the breakdown of perturbative factorization for the cross section of a physical process does not render the process useless. Factorization of the cross section for massive lepton-pair production, the Drell-Yan process, fails at order $1 / Q^{4}$ [5],6]. Breakdown means that one may not factorize contributions proportional to $1 / Q^{4}$ into infrared-safe short-distance hard parts times well-defined long-distance matrix elements. The terms proportional to $1 / Q^{4}$ are still finite, and the Drell-Yan process remains a fine process for tests of QCD dynamics, as long as $Q^{2}$ is large enough.

In isolated photon production, when $x_{\gamma} \sim 1 /\left(1+\epsilon_{h}\right)$, the breakdown of factorization demonstrated in this paper means that the cross section cannot be factored into a sum of terms each having the form of an infrared-safe partonic hard part times a corresponding parton-to-photon fragmentation function. Nevertheless, the measured cross section is still well-behaved and finite. It becomes a task to show that one can still extract meaningful information from the data on isolated photons, even if factorization does not hold throughout phase space.

In $e^{+} e^{-}$annihilation, as long as $x_{\gamma}$ is kept less than $1 /\left(1+\epsilon_{h}\right)$, the subtraction term in Eq. (2) vanishes. The short-distance hard part $\hat{\sigma}_{e^{+} e^{-} \rightarrow q X}^{(1) i s o}$ defined in Eq. (2) is then given by the isolated partonic cross section in Eq. (9). Because $x_{1}<1$ when $x_{\gamma}<1 /\left(1+\epsilon_{h}\right)$, the $\delta(1-$ $\left.x_{1}\right)$ terms do not contribute, and the "+" prescription is not relevant. The short-distance 
hard part, $\hat{\sigma}_{e^{+} e^{-} \rightarrow q X}^{(1) i s o}$, is then finite. After the convolution over $z$ in Eq. (11), terms such as $1 /\left(1-x_{1}\right)$ and $\ln \left(1-x_{1}\right) /\left(1-x_{1}\right)$ produce contributions proportional to $\ln \left(1 / x_{\gamma}-\left(1+\epsilon_{h}\right)\right)$. These are logarithmically divergent when $x_{\gamma} \rightarrow 1 /\left(1+\epsilon_{h}\right)$, but if $x_{\gamma}$ is kept much smaller than $1 /\left(1+\epsilon_{h}\right)$, the cross section for isolated photons in $e^{+} e^{-}$annihilation is well-behaved.

The opportunity to extract good information on photon fragmentation functions is one of the reasons for the study of isolated photons in $e^{+} e^{-}$annihilation. [7, 8] Equation (11) indicates that a large value of $\epsilon_{h}$ allows a large range of values of $z$. On the other hand, a large value of $\epsilon_{h}$ leaves a small range of $x_{\gamma}$ in which a fixed-order analytical calculation can be trusted. Therefore, special care must be taken when data from $e^{+} e^{-}$annihilation are used to extract photon fragmentation functions.

For production of isolated photons at hadron-hadron colliders, the physical cross section is obtained after an integration over the momentum fractions of incoming partons. One is not free to impose a selection on $x_{\gamma}$ analogous to that in $e^{+} e^{-}$annihilation, and the integration is done throughout the part of phase space where the breakdown of factorization discussed in this Letter takes place. It is therefore not altogether straightforward to specify the precise form and magnitude of the fragmentation contribution to isolated prompt photon production in hadron-hadron collisions. More discussion of this question will be found in Ref. 团.

\section{ACKNOWLEDGEMENT}

We thank George Sterman for helpful discussions. Work in the High Energy Physics Division at Argonne National Laboratory is supported by the U.S. Department of Energy, Division of High Energy Physics, Contract W-31-109-ENG-38. The work at Iowa State University is supported in part by the U.S. Department of Energy under Grant Nos. DEFG02-87ER40731 and DE-FG02-92ER40730. 


\section{REFERENCES}

[1] J. F. Owens, Rev. Mod. Phys. 59, 465 (1987), and references therein.

[2] J. C. Collins, D. E. Soper and G. Sterman, in Perturbative Quantum Chromodynamics, ed. A. H. Mueller (World Scientific, Singapore, 1989).

[3] E. L. Berger, X. Guo and J. Qiu, Argonne report ANL-HEP-PR-94-74, hep-ph/9507428, Phys. Rev. D (in press).

[4] E. L. Berger, X. Guo and J. Qiu, Argonne report, ANL-HEP-PR-95-48 (to be published).

[5] R. Doria, J. Frenkel and J. C. Taylor, Nucl. Phys. B168, 93 (1980); C. Di'Lieto, S. Gendron, I. G. Halliday and C. T. Sachrajda, Nucl. Phys. B183, 223 (1981); F. T. Brandt, J. Frenkel and J. C. Taylor, Nucl. Phys. B312, 589 (1989).

[6] J. Qiu and G. Sterman, Nucl. Phys. B353, 137 (1991).

[7] D. Buskulic et al., ALEPH Collaboration, CERN report CERN-PPE/95-089 submitted to Z. Phys.; P. Abreu et al., DELPHI Collaboration, CERN report CERN-PPE/95-101 submitted to Z. Phys.; and references therein.

[8] Z. Kunszt and Z. Trocsanyi, Nucl. Phys. B394, 139 (1993); E. W. N. Glover and A. G. Morgan, Z. Phys. C62, 311 (1994); and references therein.

\section{FIGURE}

Fig. 1. Illustration of an isolation cone containing a parton $c$ that fragments into a $\gamma$ plus hadronic energy $E_{\text {frag }}$. In addition, a gluon enters the cone and fragments giving hadronic energy $E_{\text {parton }}$. 


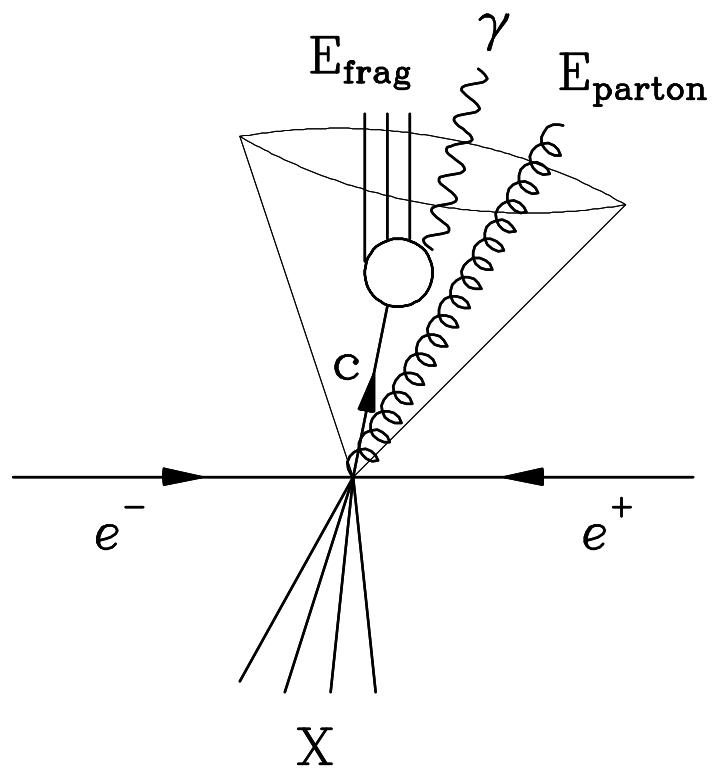

Fig.1 